\title{
Docencia y cultura bibliotecaria americana frente a la española: una experiencia
}

\author{
Por Pedro José Zapirain Sagaseta
}

Resumen: El trabajo desempeñado en la Davis Library de la "University of North Carolina at Chapel Hill" y mi formación en la "School of Information and Library Science" me ha permitido conocer la realidad de las bibliotecas y facultades universitarias americanas en la actualidad. Toda esta experiencia me da pie para contrastarla con el caso español y entresacar una serie de reflexiones y valoraciones. Los departamentos de desarrollo de la colección, las redes de bibliotecas como TRLN, la utilización del chat en los servicios de referencia y la docencia en las facultades de biblioteconomía y documentación nos permiten conocer un mundo y una cultura diferente que nos enriquece, ampliando nuestras miras, y de la que todos podemos aprender.

Palabras clave: Experiencia profesional, Desarrollo de la colec-

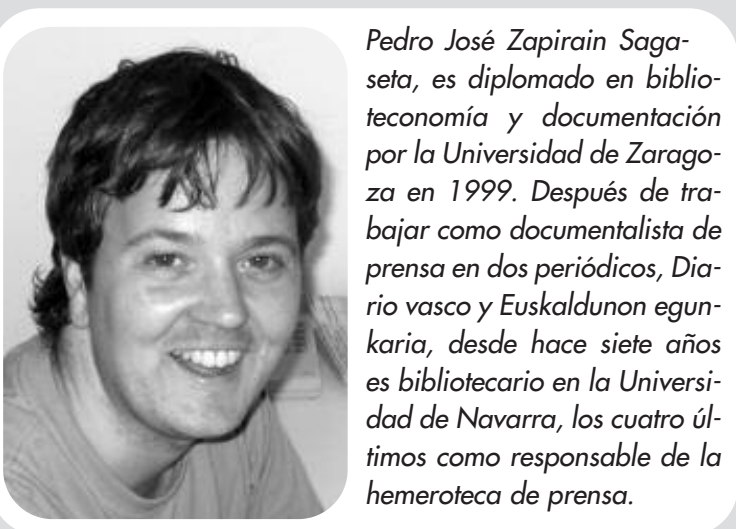

ción, Cooperación entre bibliotecas, Docencia en biblioteconomía y documentación, Servicio de chat en referencia.

Title: Comparison of Spanish and American library, teaching and culture: a personal experience

Abstract: My experience working at Davis Library at the University of North Carolina at Chapel Hill and my coursework at the School of Information and Library Science have let me learn the reality of American university libraries and academic programs. All of this insight allows me to contrast this to the Spanish context and to reflect and evaluate both from the perspective of a Spanish librarian. The collection development departments, the Triangle Research Library Network, the use of chat reference and the instruction in the School of Library and information science allow us to understand a different world and culture that enriches us and broadens our vision from which we can all learn.

Keywords: Professional experience, Collection development, Cooperation between libraries, Teaching in Library and Information Science, Chat reference services. 


\section{Introducción y contexto}

DESDE AGOSTO A DICIEMBRE DEL AÑO 2004 pasé cuatro meses en la University of North Carolina at Chapel Hill (EUA) gracias a una beca concedida por la Universidad de Navarra. Sabíamos que se trataba de un centro de gran prestigio, con una muy buena biblioteca universitaria y una oferta educativa de gran calidad, de la que teníamos noticias puesto que el Departamento de Lingüistica General y Lengua Española de nuestra universidad mantiene un intercambio con ellos desde hace casi una década.

El objetivo principal era conocer cómo se trabaja en las bibliotecas norteamericanas, colaborando estrechamente con sus profesionales en un proyecto concreto, y observar cómo se forma a los futuros bibliotecarios cursando clases de biblioteconomía y documentación en la facultad.

Además, la Universidad de $\mathrm{Na}$ varra dejó la puerta abierta para recibir un alumno que quisiera aceptar el reto de venirse a conocer y trabajar en nuestra biblioteca, algo que un poco más tarde ocurrió.

Los objetivos que nos planteamos con esta experiencia eran:

-Aprender y entender el funcionamiento de las bibliotecas americanas.

-Intuir las tendencias y orientaciones de las mismas.

—Analizar qué servicios ofrecen a sus usuarios.

-Estudiar qué proyectos y con qué tecnologías están trabajando.

- Reunir una serie de recursos de interés y un listado de contactos.

- Trasladar, en la medida de lo posible, experiencias docentes y bibliotecarias a nuestra universidad.

- Reflexionar sobre los déficits comparativos de los servicios bi- bliotecarios en España, buscar las causas y proyectar mejoras.

Las reflexiones y descripciones que trataré de reflejar en este artículo se derivan de la:

-Participación en un proyecto en el departamento de Latin American and Iberian Resources en la biblioteca principal, Davis Library, sobre el análisis de las bases de datos de prensa con las que trabajan.

-Asistencia y participación en dos clases: Information resource selection and evaluation (servicio de referencia) y Special libraries (centros de documentación y bibliotecas especializadas).

-Implicación en reuniones, entrevistas con responsables de diferentes departamentos de las diversas bibliotecas, visitas a centros de documentación y bibliotecas o asistencia a conferencias sobre biblioteconomía y documentación.

Trataré de describir y contrastar todos estos aspectos con el caso español con el objetivo de que nos aporten nuevas ideas a nuestro cambiante panorama bibliotecario.

Quisiera remarcar que no he pretendido centrarme en un estudio comparativo académico de las bibliotecas americanas y las españolas, sino esbozar un análisis de lo que mi experiencia en EUA me ha aportado desde el punto de vista de un bibliotecario español que ha vivido la experiencia en primera persona.

\section{Aspectos de la cultura bibliotecaria. Un acercamiento}

\section{Cultura bibliotecaria}

a. Departamento de desarrollo de la colección (collection development).

La biblioteca central cuenta con un departamento específico cuyo personal bibliotecario está formado y orientado hacia la consecución de estos objetivos ${ }^{1}$ :
-Favorecer el desarrollo de las colecciones al servicio de los usuarios creando políticas y buscando financiación.

- Seleccionar, evaluar y gestionar los documentos que más se ajusten a las necesidades de sus usuarios.

-Mantener criterios uniformes y preestablecidos de qué se va a adquirir, en qué formato, cuáles se van a compartir y pagar entre varias instituciones o cómo expurgar.

Todo ello irá en consonancia con sus «mission statements».

\section{«Las relaciones entre responsables de la compra de libros y los departamentos que imparten las materias son muy estrechas»}

En cada departamento el responsable máximo tiene formación universitaria -a menudo un doctorado- y conocimiento de lenguas, y experiencia docente en las materias o ámbito del que se encarga junto con la carrera de biblioteconomía. La combinación de esta formación o experiencia doble es esencial para dirigir este departamento.

Las relaciones entre responsables de la compra de libros y los departamentos que imparten esas materias son muy estrechas. En algunos casos incluso el bibliotecario ha sido doctorado o docente en ese mismo departamento.

b. Cooperación entre bibliotecas. Redes ${ }^{2}$

La Triangle Research Library Network (TRLN) la forman cuatro universidades: University of North Carolina (UNC), Duke University, North Carolina Central University $(N C C U)$ y la North Carolina State University (NCSU).

Trabajan conjuntamente desde 1933 y son bibliotecas universita- 
rias tanto públicas como privadas del estado de Carolina del Norte, las cuales comparten:

—La adquisición de recursos electrónicos de forma consensuada entre los miembros de cada una de las 10 bibliotecas que pertenecen a TRLN.

-El servicio de chat en referencia que funciona 24 horas al día, los 7 días de la semana.

-Préstamo interbibliotecario gratuito entre ellas. Cuentan con un catálogo colectivo desde el que cualquier usuario puede solicitar y recoger el mismo día cualquier documento de la red de bibliotecas enviado mediante un transporte interno por cualquiera de los otros centros.

-Desarrollo de la colección. Se reparten la adquisición de documentos en función de las carreras que se imparten y su trayectoria histórica. Mientras que, por ejemplo, UNC-Chapel Hill adquiere todo lo relativo a China, la República Checa y Eslovaquia, Duke se encarga de Japón y Polonia.

\section{«La aplicación del sistema americano implica un cambio de mentalidad y cultura, para el que quizá todavía no estemos preparados"}

Son bibliotecas especializadas, en las que la selección de fondos es muy cuidada.

\section{Tecnología. Servicio de chat en referencia ${ }^{3}$}

Hay tres bibliotecas y un proyecto a nivel estatal llamado $N C$ Knows, que desde lugares físicos distintos y valiéndose de diferentes tecnologías, trabajan sobre la misma idea: asistir y ayudar en la resolución de cualquier duda a todo ciudadano de Carolina del Norte mediante un programa de chat, esto es, darles una respuesta inmediata y online a sus necesidades, bien sea desde la biblioteca central del campus de Chapel Hill o de la biblioteca especializada o pública del condado más pequeño del estado. En cada caso se utilizan herramientas informáticas o programas diferentes. Así, Davis Library ofrece mediante dos programas de chat diferentes, Live Online Help e Instant Messenger (IM) a Librarian asistencia enfocada a satisfacer las necesidades de sus investigadores y alumnos; Health Sciences Library utiliza el programa Live Online Help; y la Undergraduate Library, biblioteca específicamente creada para asesorar en sus trabajos a los alumnos de primeros años de carrera aún no licenciados se vale del Messenger de Yahoo y de MSN para satisfacer las inquietudes de respuesta urgente de sus usuarios.

http://www.lib.unc.edu/livehelp/

http://www.lib.unc.edu/reference/i malibrariandavis.html

http://vrl-web1.lssi.com/wcscgi/ CDM.exe/uncch?SS_COMMAND= CUST_SUP\&Category $=H S L$

Este servicio se complementa con la posibilidad de escribir un mensaje de correo electrónico o llamar por teléfono al bibliotecario-referencista especializado en una materia o con la asistencia personalizada en el mismo mostrador, en el servicio denominado Face to face interview.

\section{Docencia ${ }^{4}$}

La School of Information and Library Science (SILS) ha sido reconocida recientemente como la más prestigiosa de biblioteconomía en todo el país especialmente por sus dos másters: Master of science in information science (MSIS) y Master of science in library science (MSLS), así como por su programa de doctorado (Doctoral program Ph. D.).

Cabe destacar que una parte importante del profesorado de SILS lo compone bibliotecarios o documentalistas en activo que puntual o periódicamente colaboran en la impartición de materias que dominan.

En otros muchos casos, diferentes expertos especializados en un área, materia o tecnología se encargan de dar sesiones concretas en cada clase a los alumnos. Las sesiones son prácticas, con apoyo en la tecnología más puntera y habitualmente con la posibilidad de que podamos seguir la clase desde nuestro ordenador mientras el ponente se conecta a una base de datos o explica una diapositiva. Casi siempre se nos adjunta una página web o pathfinder ${ }^{5}$ creado para la clase y bibliografía impresa junto con el correo y teléfono del experto.

En ocasiones nos encontramos entre los docentes de SILS antiguos o actuales bibliotecarios y también se dan casos de bibliotecarios expertos en una materia como estadística que pasan a ser profesores de la Facultad de Economía.

\section{Aplicación de estas ideas a la realidad española}

Ambos países en general cuentan con una cultura muy diferente: se dan acciones, formas de trabajar y rutinas que "encajan" y tienen sentido en un lugar con unas características y un contexto en el que son razonables y lógicas.

Sin embargo, a pesar de estas diferencias sociales, culturales y en algunos casos de desarrollo tecnológico, gran parte de los razonamientos, ideas y propuestas americanas son trasladables al ámbito bibliotecario español. Además nos aportan nuevas e interesantes ideas.

1. Cultura bibliotecaria. Depto. de Desarrollo de la Colección (collection development)

Hace más de 40 años las bibliotecas americanas asumieron poco a poco que los profesores de departamentos, quienes tenían el poder de 


\section{Nature Publishing Group}

\section{La excelencia cientifica en papel y en línea}

Nature Publishing Group (NPG) es un editor lider de información científica y médica con un programa editorial que combina la excelencia e innovación con una cobertura completa de la ciencia moderna.

\section{Publicaciones nuevas:}

- Archivo de Nature: 1950-1986

- Colección de archivos de las revistas de investigación de Nature

- Journal of Cerebral Blood Flow \& Metabolism

- Nature Chemical Biology

- Nature Methods

- Nature Physics

\section{nature \\ CLINICAL PRACTICE}

Nuevo en el 2005

- Endocrinologia y Metabolismo

- Nefrología

- Neurología

- Reumatologia

Especabidades arouncres

- Medicina cardiovascular

- Gastroenterologia y Hepatología

- Oncología

- Urología

\section{T: +44 (0)20 78434759 E: institutions@nature.com | W: npg.nature.com/libraries}

decisión y los medios económicos no tenían ni el tiempo suficiente ni la formación necesaria para encargarse ellos solos de la adquisición de todos los materiales de su área, y como consecuencia del desarrollo de la colección, dando por hecho que: "the library can be a proactive force if the faculty trust the librarians to make appropriate choices and the librarians trust the faculty to match out for institutional well being".

Partiendo de ese punto, los bibliotecarios americanos iniciaron un plan de formación para especializarse en un área temática y se pusieron los medios para la creación de un departamento especifico (Desarrollo de la colección) en el que un conjunto de personas formadas adecuadamente, con una carrera en esa especialidad, con estudios y experiencia de biblioteconomía, deciden qué documentos y materiales satisfacen mejor las necesidades de sus usuarios.

En nuestro país, por lo general y salvo contadas excepciones, los bibliotecarios no asumimos un papel importante en la adquisición de documentos en las bibliotecas universitarias. La capacidad de decisión del personal bibliotecario sobre el desarrollo de la colección es reducida y en algunos casos muy limitada.

En los EUA, los departamentos de adquisiciones acostumbran a tramitar las peticiones de compras de documentos de un departamento de una facultad, que individualmente o de modo consensuado con sus colegas decide qué recursos se van a adquirir. En otros casos, comisiones formadas por bibliotecarios y departamentos de profesores deciden colectivamente el material a incorporar a la biblioteca y que va a ser el más apropiado para los alumnos en sus clases.
La aplicación del sistema americano implica un cambio de mentalidad y cultura, para el que probablemente todavía no estemos preparados. Sin embargo, parece lógico y evidente que un acercamiento a estas posturas nos ofrecería una serie de ventajas que tendrían como puntos fuertes:

- Trabajar en un desarrollo de la colección más armonioso y equitativo buscando reunir toda la literatura científica posible de cada campo del saber sin dejar materias y disciplinas sin cubrir, esto es, sin crear lagunas en la colección.

- Buscar un diálogo activo entre departamentos de facultades y bibliotecarios para tomar decisiones sobre las adquisiciones, en el que el profesor plantea sus necesidades a su colega bibliotecario, también experto. El bibliotecario, al ser conocedor del mundo bibliográfico tiene la última palabra para de- 
cidir qué documentos y en qué formato se van a incorporar.

- Analizar y evaluar constantemente nuestro desarrollo de la colección, la pertinencia, exhaustividad y utilidad de los fondos y paliar de modo automático los desajustes que se pudieran dar.

a. Cooperación entre bibliotecas. Redes $(T R L N)$ y recursos compartidos.

La TRLN es todo un ejemplo de cooperación, por lo estrecha y duradera en el tiempo, habitual entre bibliotecas americanas, pero no sólo entre las bibliotecas universitarias públicas de un mismo estado, sino también con universidades privadas como la Duke University.

Por otro lado, hemos comprobado que han tomado iniciativas tan interesantes como NC Live mediante las cuales cualquier ciudadano que sea usuario de una biblioteca de Carolina del Norte, sólo con el número del carné y desde cualquier PC conectado a la Red tiene la posibilidad de acceder gratuitamente a miles de recursos de interés como periódicos, revistas, enciclopedias, índices, etc. .

Encajando perfectamente con una cultura bibliotecaria de mentalidad abierta, he detectado que se funciona a todos los niveles respetando unos procedimientos que seguirían el siguiente esquema: como consecuencia de un trabajo en equipo, con un presupuesto que se ha buscado a partir de un proyecto concreto que se ha sabido vender a un departamento, institución pública o privada o a un particular, se realiza una labor que da como fruto una recopilación de información de interés para la comunidad y disponible indefinidamente para todo el mundo en una página web pública ${ }^{8}$.

En España tenemos un amplio abanico de iniciativas de cooperación que han dado lugar a redes, consorcios, creación de catálogos colectivos y protocolos que han logrado que nos unamos para compartir e intercambiar lo que en cada institución tenemos.

\section{«El usuario aprende mientras busca, trabaja, investiga, por lo que las clases estrictamente teóricas tienen menor carga y son menos densas»}

Sin embargo, sigue dando la impresión de que nuestras iniciativas son todavía tímidas, abarcan un espectro reducido de las labores bibliotecarias que pueden ser compartidas y crecen a un ritmo más lento del deseable. En algunos casos, las cooperaciones se dan aprovechando las relaciones lógicas entre la biblioteca central y las sucursales de una misma comunidad, pero quizás debamos avanzar hacia una cooperación más abierta y flexible:

— ¿Qué supondría si cada provincia española tuviera acuerdos de cooperación con las instituciones que recogen y conservan información (del tipo que sea) de su misma provincia o con las mas cercanas?

— ¿Qué ventajas obtendríamos si todo tipo de bibliotecas, archivos, museos y centros de documentación de una provincia o comunidad autónoma nos repartiéramos la adquisición de aquellos documentos que nos interesan a todos? Una vez garantizáramos al usuario el acceso a la información, bien mediante un sistema de préstamo interbibliotecario ágil y efectivo o bien permitiendo el acceso al documento primario en cada centro aunaríamos eficiencia con bajo coste.

- ¿Qué representaría que todos o parte de los recursos electrónicos pudieran ser accesibles desde cualquier punto de esta nueva "red de centros de investigación" por cualquier usuario, tanto interno como externo?
- ¿Qué implicaría, una vez desarrollada esta primera fase, unirnos todas las instituciones y crear redes a todos los niveles: comunitario, estatal, etc.?

La mera idea, lejos de ser especialmente novedosa, llevada a sus máximas consecuencias representaría un antes y un después en nuestros intentos de darnos a conocer y cooperar, un ámbito en el que desde el primer momento me dio la sensación de que en EUA han dado pasos más firmes y decididos.

\section{Tecnología. Servicio de chat} en referencia

No cabe duda de que las nuevas tecnologías se van incorporando a nuestra vida de un modo progresivo e incesante y que han incidido en el panorama de las bibliotecas de un modo más o menos decisivo.

Los servicios de referencia de las bibliotecas universitarias españolas cuentan con varios servicios y diferentes medios para interactuar o alimentar el feedback con el usuario. Hasta el momento van siendo comunes las peticiones y consultas por correo electrónico y por teléfono a parte de la tradicional y efectiva entrevista en persona con el bibliotecario-referencista.

Más recientemente, en algunas bibliotecas americanas, como la Davis Library de UNC, se ha apostado por un nuevo servicio de "referencia digital". Se trata de un chat mediante el cual el usuario lanza una pregunta y es respondido, en cuestión de segundos, por un referencista, especializado en el tema en el que está interesado. Por lo general, se utiliza cuando el usuario se encuentra físicamente lejos de la biblioteca y tiene una duda puntual.

Esta herramienta, todavía en fase de pruebas en algunas universidades norteamericanas, es todavía bastante desconocida en España y que sepamos, aún no se ha implan- 
tado en ninguna biblioteca de nuestro país y en muy pocas en Europa.

No cabe duda de que un medio como éste, y más si funcionara "24/7" no puede plantearse sin una sólida formación del personal, unos recursos y por supuesto una planificación concienzuda, pero a nadie se le escapa que aportaría a nuestros usuarios:

—Un sistema ágil y cómodo para interactuar con los bibliotecarios especializados en referencia.

-Una gran ayuda para los investigadores, que encontrarían un cauce directo y flexible en el tiempo para resolver dudas y continuar en sus investigaciones, en cualquier lugar y momento.

-Un servicio más que complementaría los ya existentes de teléfono, correo electrónico y en persona pero aportando inmediatez, comodidad y sencillez.

-Una nueva dimensión del mundo de la información en la que el acceso y la recuperación, los canales e inmediatez de la misma abren nuevos horizontes y suponen todo un reto para el mundo bibliotecario.

Evidentemente, no podemos dejar de plantear una serie de dudas si se implantase en España como: ¿podremos atender los bibliotecarios todas las preguntas?, ¿no se convertirá en un servicio que nos desborde?, ¿debemos dedicar personal altamente especializado exclusivamente a este servicio?, ¿será un servicio realmente útil para nuestros usuarios?

\section{Docencia. Clases en SILS}

La docencia en las universidades anglosajonas, y en concreto en las americanas, y sobre todo en carreras tan prácticas como biblioteconomía y documentación funciona con una lógica, unas dinámicas y un modo de proceder muy alejados de los esquemas españoles tradiciona- les. Una vez mas la cultura, el desarrollo tecnológico, los medios y aspectos como un número reducido de alumnos por clase son básicos para entender el enfoque norteamericano.

Bien es cierto que en los últimos años la educación tanto en nuestro país como en el resto de Europa está sumida en una continua revisión y afectada por permanentes y numerosos cambios y transformaciones ${ }^{10}$. Sin embargo, parece que el modelo americano presenta características algo alejadas del sistema educativo universitario en España:

a. Clases eminentemente prácticas.

Con obligatoriedad de realizar trabajos de investigación en grupos, lecturas y presentaciones en el aula. La idea es que el alumno utilice y desarrolle sus recursos y destrezas para buscar información y conocer las fuentes. Los estudiantes recurren a la biblioteca, hablan con el referencista, buscan revistas online, se suscriben a listas de distribución y acuden a entrevistar a bibliotecarios para los trabajos en clase. Las presentaciones (en algunos casos representaciones) son individuales o grupales y al alumno que escucha en clase se le exige atender, preguntar y se le alienta para que aporte críticas constructivas.

Por lo general se da por hecho que:

-El usuario aprende mientras busca, trabaja, investiga, por lo que las clases estrictamente teóricas tienen menor carga y son menos densas.

—El profesor no es una "figura omnipotente" que te conduce a la verdad, sino una persona de apoyo que te guía en tu propio conocimiento.

—Lo más importante para el profesor es que el alumno interiorice el hábito de trabajar semanal- mente en proyectos individuales o coordinadamente en grupo, respetando unas normas y procedimientos. En muchos casos no existe el examen final.

-El conocimiento de uno tiene que ser compartido siempre y estar disponible en todo momento para todos. Para ello se enviarán los trabajos, conclusiones extraídas en foros y los proyectos en los que se ha trabajado al resto de alumnos y al profesor.

b. Las clases están abiertas y a veces pensadas para graduados en otras carreras.

Las clases en la facultad de "ciencias de las bibliotecas" buscan que avancen en sus conocimientos alumnos con formación en biblioteconomía y documentación, que se actualicen y reciclen los bibliotecarios o archiveros profesionales o se especialicen y orienten los licenciados en otras carreras como: economía, literatura, ciencias de la salud, etc. Este último colectivo es especialmente interesante y requerido para trabajar como bibliotecario especialista en su materia en departamentos como: referencia, catalogación o desarrollo de la colección.

c. Compaginar estudio con trabajo.

La gran mayoría de los alumnos, o son profesionales en activo o compaginan sus trres o cuatro clases por cuatrimestre con un trabajo remunerado, normalmente a media jornada en alguna biblioteca. De este modo los conocimientos que se van adquiriendo se complementan con su aportación en un proyecto específico para un departamento.

d. Interacción entre profesores y bibliotecarios.

Las clases están presentadas muy a menudo por expertos en un área temática o especialidad que dan una visión general de su trabajo diario y aportan sus conocimientos y experiencia ante unos alum- 
nos interesados en conocer y aplicar esos conocimientos a su trabajo o a proyectos de investigación.

\section{Conclusiones}

El hecho de que el servicio de bibliotecas de una universidad plantee en un contexto de formación de sus empleados que dediquen e inviertan unos tres o cuatro meses a conocer como observadores activos la "gestión de la información" en diferentes bibliotecas de otros países representa un punto de partida tan interesante como infrecuente en nuestro panorama bibliotecario.

Animamos a las instituciones de nuestro país a crear vínculos y a fomentar intercambios del personal bibliotecario y relaciones con otras bibliotecas extranjeras.

Gran parte de los aspectos que hemos descrito son conocidos en España pero presentan grandes diferencias, tanto de planteamiento como de cultura, frente a las gestiones y proyectos en los que estamos involucrados en nuestro día a día.

La creación de redes de bibliotecas en estados como el de Carolina del Norte, habla mucho y muy bien de la cooperación estrecha que se da entre diferentes centros de todo tipo, universitarios (públicos y privados), especializados y públicos.

La existencia de un departamento propio de "desarrollo de la colección" nos abre los ojos ante un panorama en el que el bibliotecario, con un perfil altamente especializado, tiene control y autoridad frente a las adquisiciones en un mundo en el que cada vez más, este profesional tiene que ser capaz de asumir más responsabilidades y representar un papel más influyente en la comunidad universitaria.

Recurrir a técnicas como el chat para resolver dudas bibliográficas de nuestros usuarios se presenta como una herramienta ágil, e inme- diata que probablemente termine siendo un servicio que añadiremos a los que ya estábamos prestando hasta el momento también en nuestras bibliotecas.

El sistema educativo americano, con sus pros y contras, tiene características que lo definen y diferencian de nuestras facultades de biblioteconomía y documentación. Creo que en España nos estamos acercando hacia un modelo semejante al americano que, sin embargo, por su enfoque práctico -tan encaminado a la experimentación y a la investigación, orientado tanto a especializar a gente de otras carreras como a formar a técnicos en activo- representa una idea que, en su conjunto, guía mejor en el conocimiento del trabajo y desempeño de las funciones del profesional de la información. Esto es, se acerca mucho más a la realidad con la que luego se encuentran los profesionales de las bibliotecas.

Los conocimientos teóricos son básicos y tienen una vital importancia en la toma de decisiones y como soporte para adaptarnos a los cambios, pero en el caso americano entienden que pueden ser adquiridos a partir del trabajo práctico, y llevan esta premisa hasta sus últimas consecuencias.

A pesar de un sinfín de diferencias más o menos relevantes, los especialistas en gestionar, tratar y difundir la información pretendemos satisfacer las necesidades de un usuario final al que nos debemos, y que ha de estar siempre en nuestro "punto de mira", y ahí es donde los bibliotecarios, sin fronteras, tenemos que coincidir.

El camino puede ser múltiple, pero si contamos con el apoyo y cooperación mutua, el final se presenta menos oscuro y más asequible.

\section{Notas}

1. Página explicativa del departamento de $\mathrm{Co}$ llection Development.

http://www.lib.unc.edu/cdd/index.html
2. http://www.trln.org/

3. Página web sobre los diferentes servicios de chat que ofrece $U N C$.

http://www.lib.unc.edu/chat.html

4. http://sils.unc.edu/index.htm

5. Según M. Reitz, Joan: "The dictionary for library and information science" (2004) lo define como: "a subject bibliography designed to lead the user through the process of researching a specific topic, or any topic in a given field or discipline, usually in a systematic step-by-step way, making use of the best finding tools the library has to offer. Pathfinders may be printed or available online".

Sirva como ejemplo el pathfinder creado por el departamento de Latin American and Iberian Resources para guiar al usuario en sus investigaciones sobre el cine latinoamericano.

http://www.lib.unc.edu/cdd/crs/international/lati n/pathfinder/cinema/index.html

6. Ochu, Felix T. «Librarian-faculty relations in collection development". En: The journal of academic librarianship, 1997, enero, p. 17.

7. North Carolina Libraries for Virtual Education (NC Live) offers the citizens of North Carolina online access to complete articles from over 10.000 newspapers, journals, magazines, and encyclopedias, indexing for over 20.000 periodical titles, and access to over 24.000 electronic books. NC Live is available free of charge to library patrons, students, and educators from four communities of interest (COIs)-public libraries, community colleges, the state's university system, and members of the North Carolina Association of Independent Colleges and Universities. NC Live can be accessed from within an affiliated library or from home.

Texto completo en:

http://www.nclive.org/about.phtml

8. Como proyectos en los que está involucrada la University of North Carolina at Chapel Hill con este espíritu destacamos dos:

Open Video Project. Miles de documentales sobre América del Sur.

http://www.open-video.org/

Documenting the American South. Cientos de documentos digitalizados a texto completo y grabaciones sonoras sobre la historia del sur de América.

http://docsouth.unc.edu/

9. Página de la IFLA que define las diferentes acepciones y da a conocer algunas recomendaciones sobre la administración y la puesta en práctica de un servicio de chat en una biblioteca. http://www.ifla.org/VII/s36/pubs/drg03-s.htm\#2.3

10. A este respecto cabe destacar la Declaración de Bolonia como iniciativa que en muchos aspectos toma la senda o traza su propio camino pero con un espíritu que creemos se acerca al de las universidades americanas actuales.

Pedro José Zapirain Sagaseta, Universidad de Navarra, Servicio de Bibliotecas-Hemeroteca, Apdo. 177, 31080, Pamplona, España. pzapirain@unav.es http://www.unav.es/biblioteca/huhemdefinicion.html 


\section{C $i$ Nuenta años GESTIONANDO DOUumentación CIENTIFICA}

\section{Bases de Datos CSIC Lo nuestro son los CONTENIDOS}

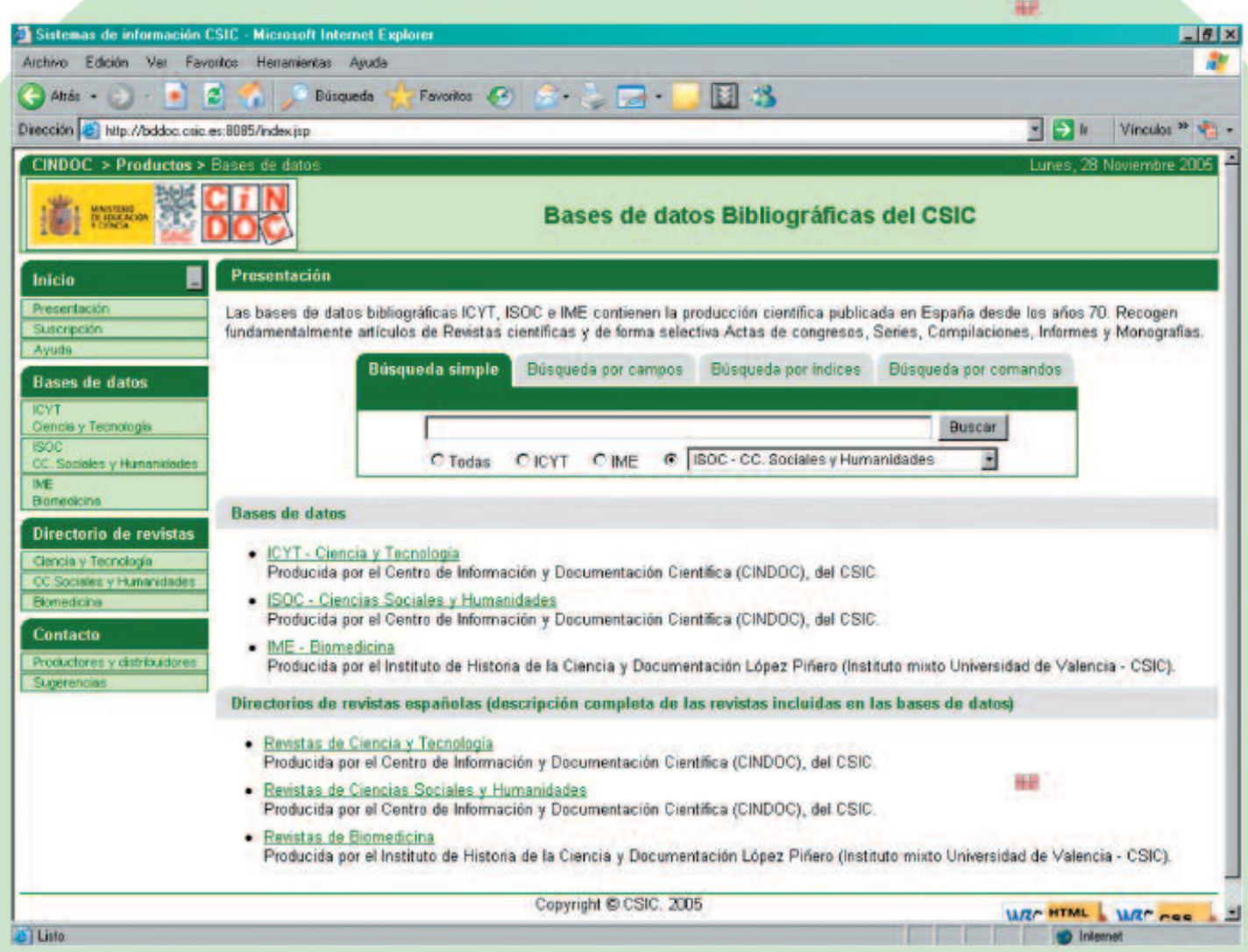

\section{http://www.cindoc.csic.es}

